\title{
O percurso da avaliação psicológica nos Simpósios da ANPEPP
}

\author{
Elizabeth do Nascimento ${ }^{1}$, Alina Gomide Vasconcelos \\ Universidade Federal de Minas Gerais, Belo Horizonte-MG, Brasil
}

\section{RESUMO}

Será apresentado, em uma perspectiva histórica, a participação da área da avaliação psicológica (AP) nas edições do Simpósio Bienal de Pesquisa e Intercâmbio Científico da Associação Nacional de Pesquisa e Pós-Graduação em Psicologia (ANPEPP). Tais Simpósios constituem em uma das principais ações dessa Associação, contribuindo para o intercâmbio e a cooperação entre os centros de pesquisa e pesquisadores. Constatou-se a presença da AP desde a primeira edição do simpósio, ocorrido em 1988. Enquanto, nas décadas de 1990 e 2000, um único grupo de trabalho (GT) reunia pesquisadores de AP, na virada da década passada para a atual, o número de GTs de AP aumentou de três para cinco. A criação desses novos GTs reflete a expansão dessa área no âmbito da pesquisa, tanto pelo número de pesquisadores envolvidos quanto pela diversidade de temas relacionados à AP. Produções científicas advindas dos GTs têm contribuído substantivamente para o desenvolvimento da Psicologia no Brasil.

Palavras-chave: avaliação psicológica; ANPEPP; intercâmbio científico.

\section{ABSTRACT - The path of psychological assessment in ANPEPP Symposiums}

We present here, in a historical perspective, the participation of area of psychological assessment (PA) in the editions of the Biennial Symposium on Research and Scientific Exchange of the National Association for Research and Graduate Studies in Psychology (ANPEPP). These Symposiums constitute one of the primary actions of this Association, contributing to the exchange and cooperation between research centers and researchers. The presence of PA dates back to the first edition of the symposium in 1988. In the 1990s and 2000s, there was only one PA work group. However, from 2000 to the present, the number of work groups increased from three to five. The creation of these additional work groups reflects the expansion of the area under research, both in number of researchers involved and in the diversity of issues related to PA. Scientific production resulting from these work groups has contributed substantially to the development of psychology in Brazil.

Keywords: psychological assessment; ANPEPP; scientific exchange.

\section{RESUMEN - La ruta de evaluación psicológica en Simposios ANPEPP}

Presentamos en una perspectiva histórica la participación del área de Evaluación Psicológica (EP) en las ediciones del Simposio Bienal de Asociación Nacional de Investigaciones y Postgrado en Psicología (ANPEPP). Tales Simposios se constituyen en una de las principales acciones de esta Asociación, que contribuyen para intercambio y cooperación entre centros de investigación e investigadores. Se constató la presencia de la EP desde la primera edición del Simposio en 1988. En las década de 1990 a 2000 , un solo grupo de trabajo (GT) reunió investigadores de EP. Desde el comienzo del año 2000 hasta la actualidad, el número de GTs aumentó de tres a cinco. La creación de estos nuevos GTs refleja la expansión de esa área en el ámbito de la investigación, tanto por el número de investigadores como por la diversidad de temas relacionados con la EP. Las producciones científicas provenientes de los GTs han contribuído sustancialmente para el desarrollo de la Psicología en Brasil.

Palabras clave: Evaluación psicológica, ANPEPP, intercambio científico.

A Associação Nacional de Pesquisa e Pós-Graduação em Psicologia (ANPEPP) foi fundada em 1983 e, de acordo com seu estatuto, possui como objetivo social reunir os programas de pós-graduação (PPGs) em Psicologia vinculados a instituições de ensino superior. Atualmente, a ANPEPP reúne 71 programas de diferentes regiões do Brasil. Tal congregação possui vários objetivos específicos, dentre eles, estimular a formação de profissionais para pesquisa e pós-graduação e incentivar o aperfeiçoamento dos programas de seus associados por meio de intercâmbio e cooperação entre os centros de pesquisa e seus pesquisadores. Além disso, visa contribuir para o desenvolvimento e o fortalecimento da ciência e da profissão de psicólogo por meio da divulgação dos trabalhos científicos em Psicologia e colaborar com outras sociedades científicas na defesa dos interesses 
nacionais, especialmente, com relação à pesquisa e à pós-graduação em Psicologia. A partir de uma recomendação do Comitê Assessor do Conselho Nacional de Desenvolvimento Científico e Tecnológico (CNPq) da década de 1980 (formado pela Angela Biaggio, Terezinha Carraher e Timothy Mulholland), a ANPEPP se dispôs a realizar um encontro que permitisse avaliar a contribuição científica dos programas de pós-graduação em Psicologia (Schliemman \& Falcão, 1988).

Desde então, destaca-se a realização dos Simpósios Bienais de Pesquisa e Intercâmbio Científico. Durante o período de 1988 e 1990, esses simpósios foram anuais, adotando-se o caráter de bienal em seguida. A seguir, será apresentada, em uma perspectiva histórica, a participação da área da Avaliação Psicológica (AP) nas edições do simpósio. O conhecimento desse percurso é importante no sentido de enfatizar a consolidação dos estudos realizados na pós-graduação e a ampliação das discussões entre os pesquisadores brasileiros sobre as especificidades e implicações técnicas e éticas da AP.

A primeira edição do simpósio da ANPEPP aconteceu em Caruaru/PE, no ano de 1988, e foi organizado pelos pesquisadores Analúcia Dias Schliemman e Jorge Tarcísio R. Falcão. Nessa edição, cada PPG pôde enviar três professores para representá-lo (Schliemman \& Falcão, 1988). O formato da primeira edição seguiu os padrões convencionais de um simpósio nos quais a programação das atividades foi organizada em torno de apresentações de pesquisadores sobre temas em Psicologia em que eram especialistas. A seleção dos trabalhos apresentados buscou assegurar a participação de todos os PPGs por meio de indicações de pesquisadores (Schliemman \& Falcão, 1988). Nos anais do primeiro simpósio, dentre as apresentações elencadas, observa-se que duas delas se referiram explicitamente a temas da área da AP, a saber: "A prova de Rorschach, a epilepsia temporal e a especialização hemisférica", apresentada pela Professora Latife Yazigi e "O desenho infantil como instrumento de avaliação do desenvolvimento cognitivo, afetivo e psicomotor: descrição de uma linha de pesquisa", apresentada pelo Professor Claudio Hutz (Schliemman \& Falcão, 1988).

O I Simpósio foi um marco importante na história da Psicologia como um espaço comum para discussão de temas que interessavam a todos os pesquisadores psicólogos. Se por um lado explicitou a diversidade de métodos, perspectivas epistemológicas e relações entre clínica, pesquisa e prática em Psicologia; por outro lado, permitiu constatar que a maioria das linhas de pesquisa sobre temas comuns (com perspectivas semelhantes ou alternativas) se desenvolvia isoladamente nos PPGs sem intercâmbio entre os trabalhos desenvolvidos por pesquisadores brasileiros (Camino \& Schliemman, 1988). Como recomendação para o II Simpósio, sugeriu-se que fosse incluída a atividade de grupos de trabalho (GT), com a proposta de reunir pesquisadores segundo temas de interesse e áreas de conhecimento para debater os resultados dos estudos (Camino \& Schliemman, 1988).

Assim, no II Simpósio da ANPEPP (Gramado/RS), surgiu o primeiro GT cujo tema central foi Avaliação Psicológica e foi denominado "Perspectivas de Avaliação e Diagnóstico em Psicologia", composto por nove participantes, sendo eles, Odete L. VanKolck, André Jaquemin, Claudio Hutz, Eda M. Custódio, Edwiges Silvares, Nilce Mejias, Edwiges Silvares, Latife Yazigi, Regina M. L. L. Caravalho e Antônio Carelli. É interessante observar que esse GT foi então constituído por pesquisadores de São Paulo, com exceção do professor Claudio Hutz (ANPEPP, 1989). Já nesse primeiro momento, discutiu-se a complexidade e abrangência da Avaliação Psicológica, tendo em vista aspectos metodológicos e abordagens distintos a partir de apresentação de casos clínicos até o levantamento de dados epidemiológicos (ANPEPP, 1989). Tal GT permaneceu na terceira edição que aconteceu em Águas de São Pedro/SP (Franco \& Macedo, 1990).

A partir da quarta (1992) e quinta (1994) edições, os simpósios da ANPPEP passaram a ser temáticos. No entanto, nos anais dessas duas edições, não consta nenhum GT cujo tema abordava explicitamente a Avaliação Psicológica (ANPEPP, 1992; 1994). Já no VI Simpósio (Teresópolis/RJ, 1996), foi realizado o GT "Interação, cultura e personalidade: contribuição das técnicas projetivas", composto de quatro pesquisadores (ANPEPP, 1996).

No VII Simpósio (1998), realizado na cidade de Gramado/RS, o GT "Pesquisa em Avaliação Psicológica" foi proposto pela primeira vez, sendo coordenado pela professora Solange Wechsler (Wechsler, 1998a; Wechsler, 1998b). Esse mesmo GT, ainda que com variações na sua composição, esteve presente nas quatro edições subsequentes (VIII Simpósio, Serra Negra/SP-2000, IX Simpósio, Águas de Lindóia/SP-2002, X Simpósio, Aracruz/BA-2004 e XI Simpósio, Florianópolis/SC2006) (Boruchovitch \& Nascimento, 2012; Hutz, 2002; Weschler, 2000).

É importante ressaltar que, foi na oitava edição (2000) que se decidiu pela criação do Instituto Brasileiro de Avaliação e Pesquisa em Psicologia (IBAP), em prol do desenvolvimento da Psicologia brasileira por meio da pesquisa em Avaliação Psicológica (Hutz, 2002). Outras duas ações importantes são frutos também desse grupo de trabalho, sendo elas, a realização do I Congresso Nacional de Avaliação Psicológica (juntamente com o VII Congresso de Avaliação Psicológica no Rio Grande do Sul em parceria com o Conselho Regional de Psicologia) e a criação de uma rede de internet denominada AvalPsi, que contribui para o intercâmbio de informações entre centenas de pesquisadores, profissionais e estudantes (Hutz, 2002).

Durante o XI Simpósio (Florianópolis/RS-2006), efetivou-se a reorganização do GT "Pesquisa em 
Avaliação Psicológica” em três novos grupos em decorrência do aumento expressivo no número de pesquisadores interessados em participar (cerca de 30 participantes) e da diversidade de temáticas (Boruchovitch \& Nascimento, 2012). Esses GTs se reuniram pela primeira vez na XII edição (Natal/RN-2008). A seguir, uma breve descrição de cada um deles: 1. o GT "Pesquisa em Avaliação Psicológica", que serviu de matriz para as primeiras discussões sobre a pesquisa na área, foi mantido e composto por pesquisadores interessados em diversos construtos e contextos da área de Avaliação Psicológica; 2. o GT "Métodos projetivos em avaliação Psicológica" foi criado para oferecer respaldo aos profissionais quanto às dificuldades relativas à cientificidade e credibilidade dos testes psicológicos projetivos, bem como a carência de investigações científicas sistematizadas na área, sobretudo no Brasil; 3. o GT "Avaliação psicológica de crianças e adolescentes" foi criado para desenvolver e validar instrumentos e técnicas especialmente para a Avaliação Psicológica e Neuropsicológica de crianças e adolescentes (Alves, 2008; Boruchovitch \& Nascimento, 2012). A constituição desses três GTs permitiu articular diferentes focos de interesse na área da Avaliação Psicológica (Boruchovitch \& Nascimento, 2012).

Tais GTs se mantiveram na décima terceira (2010) e décima quarta (2012) edições dos Simpósios
(Boruchovitch \& Nascimento, 2012; Santos, 2010). Novamente, o aumento no número de membros acarretou na necessidade do estabelecimento de novos GTs, de acordo com as áreas de expertise dos pesquisadores. Por isso, na edição mais recente, XV Simpósio (2014), observou-se a participação dos dois GTs já estabelecidos (GT "Pesquisa em Avaliação Psicológica" e GT "Métodos projetivos nos contextos da Avaliação Psicológica") e a subdivisão do GT "Avaliação psicológica de crianças e adolescentes" em três novos grupos com temáticas específicas, a saber: 1. "Avaliação Cognitiva e Neuropsicológica", 2. "Avaliação em Psicologia Positiva e Criatividade" e 3. "Avaliação psicológica: personalidade e psicopatologia”. Logo, na última edição, dentre os 69 GTs inscritos no Simpósio, cinco trataram de diferentes aspectos da avaliação psicológica (ANPEPP, 2014).

A criação dos novos GTs explicita o crescimento da área da Avaliação Psicológica no país, tal como pode ser verificado na Figura 1. A organização desses grupos tem como perspectiva viabilizar pesquisas e produção científicas conjuntas que possam contribuir substantivamente para o desenvolvimento da Psicologia no Brasil.

As listas de membros dos respectivos GTs encontram-se disponíveis na página do último Simpósio da ANPEPP (http://www.anpepp.org.br/). O próximo simpósio será realizado em 2016 em Maceió/AL.

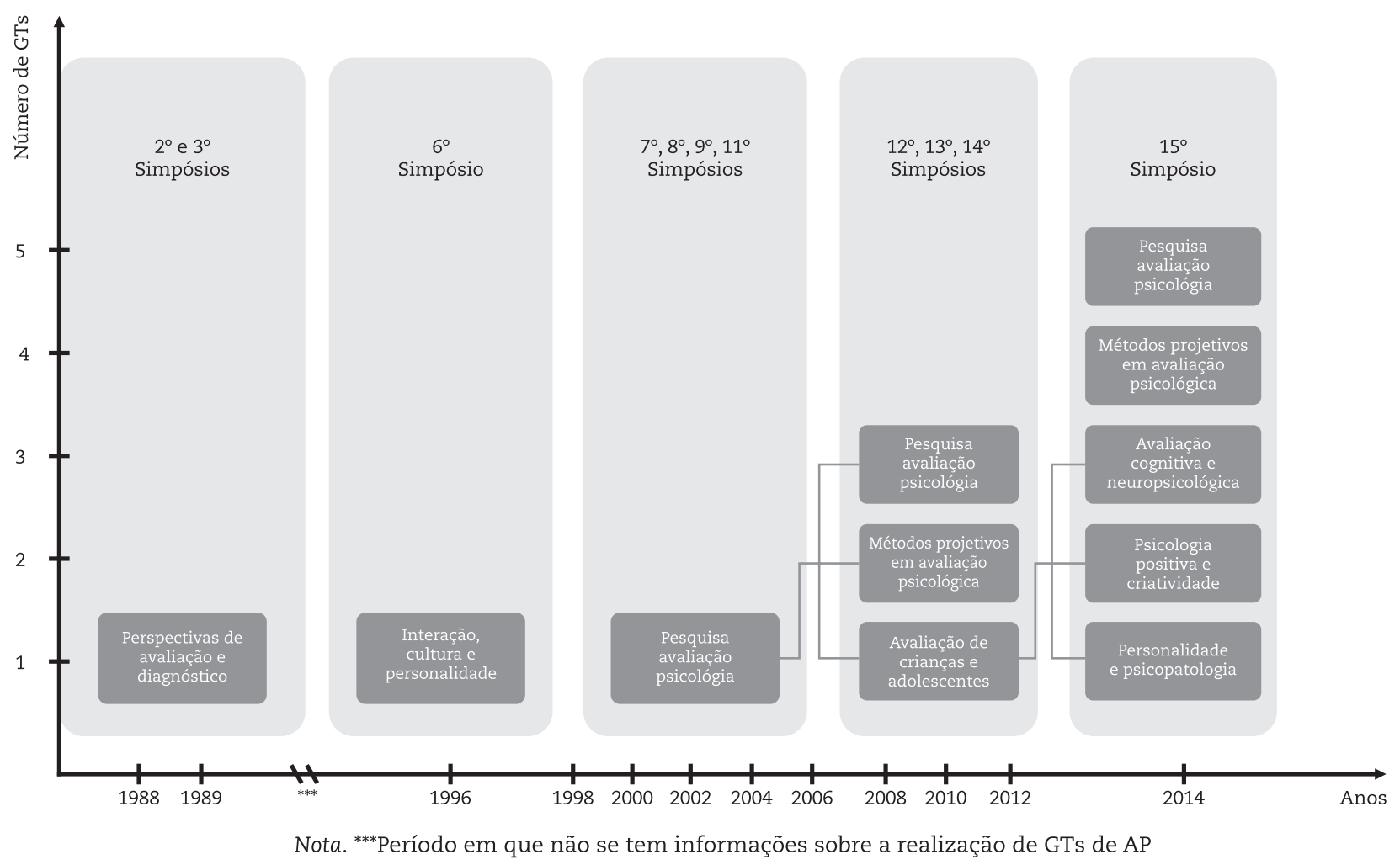

Figura 1. Frequência de grupos de trabalho em Avaliação Psicológica nos simpósios da ANPEPP (1988 - 2014) 


\section{Referências}

Alves, I. C. B. (2008). Pesquisa em avaliação psicológica, grupo de trabalho. Em Associação Nacional de Pesquisa e Pós-Graduação em Psicologia (Eds.), Anais, XII simpósio de pesquisa e intercâmbio científico: Conhecimento em psicologia no Brasil - expansão e avaliação (p. 112 115). Natal, RN: ANPEPP.

Associação Nacional de Pesquisa e Pós-Graduação em Psicologia [ANPEPP] (Eds.) (1989). Anais, II Simpósio de Pesquisa e Intercâmbio Científico. Gramado, RS: ANPEPP.

Associação Nacional de Pesquisa e Pós-Graduação em Psicologia [ANPEPP] (Eds.) (1992). Anais, IV Simpósio de Pesquisa e Intercâmbio Científico. Brasília, DF: ANPEPP.

Associação Nacional de Pesquisa e Pós-Graduação em Psicologia [ANPEPP] (Eds.) (1994). Anais, VI Simpósio de Pesquisa e Intercâmbio Cientifico. Caxambu, MG: ANPEPP.

Associação Nacional de Pesquisa e Pós-Graduação em Psicologia [ANPEPP] (Eds.) (1996). Anais, VII Simpósio de Pesquisa e Intercâmbio Científico. Teresópolis, RJ: ANPEPP.

Associação Nacional de Pesquisa e Pós-Graduação em Psicologia [ANPEPP] (Eds.) (2014). Anais, XV Simpósio de Pesquisa e Intercâmbio Científico: Pesquisa, colaboração e comprometimento em Psicologia. Bento Gonçalves, RS: ANPEPP.

Boruchovitch, E., \& Nascimento, E. (2012). Pesquisa em avaliação psicológica, grupo de trabalho. Em Associação Nacional de Pesquisa e Pós-Graduação em Psicologia (Eds.), Anais, XIV simpósio de pesquisa e intercâmbio científico - produção e divulgação científica: Os desafios da interdisciplinaridade (p. 133 - 137). Belo Horizonte, MG: ANPEPP.

Camino, L., \& Schliemman, A. D. (1988). Avaliação geral dos trabalhos apresentados no I Simpósio Brasileiro de Pesquisa e Intercâmbio Científico da ANPEPP: como vai a pesquisa em Psicologia no Brasil? Em Associação Nacional de Pesquisa e Pós-Graduação em Psicologia (Eds.), Anais, I Simpósio de Pesquisa e Intercâmbio Científico (p. 275-278). Caruaru, PE: ANPEPP.

Franco, M. L. P. B., \& Macedo, R. M. S. (1990). Apresentação. Em Associação Nacional de Pesquisa e Pós-Graduação em Psicologia (Eds.), Anais, III Simpósio de Pesquisa e Intercâmbio Científico (p. III-V). Águas de São Pedro, SP: ANPEPP.

Hutz, C. S. (2002). Pesquisa em avaliação psicológica, grupo de trabalho. Em Associação Nacional de Pesquisa e Pós-Graduação em Psicologia (Eds.), Anais, IX Simpósio de Pesquisa e Intercâmbio Científico (p. 79-89). Águas de Lindoia, SP: ANPEPP.

Santos, A. A. A. (2010). Pesquisa em avaliação psicológica, Grupo de trabalho. Em Associação Nacional de Pesquisa e Pós-Graduação em Psicologia (Eds.), Anais, XIII Simpósio de Pesquisa e Intercâmbio Científico: pesquisa em Psicologia - formação, produção e intervenção (p. 78 - 80). Fortaleza, CE: ANPEPP.

Schliemman, A. D., \& Falcão, J. T. R. (1988). Apresentação. Em Associação Nacional de Pesquisa e Pós-Graduação em Psicologia (Eds.), Anais, I Simpósio de Pesquisa e Intercâmbio Científico (p. 11-12). Caruaru, PE: ANPEPP.

Wechsler, S. (1998a). Pesquisa em avaliação psicológica, Grupo de trabalho. Em Associação Nacional de Pesquisa e Pós-Graduação em Psicologia (Eds.), Anais, VII Simpósio de Pesquisa e Intercâmbio Científico (p. 43-48). Gramado, RS: ANPEPP.

Wechsler, S. (1998b). Prefácio. Psicologia Escolar e Educacional, 2(2),85-85.

Weschler, S. (2000). Pesquisa em avaliação psicológica, Grupo de trabalho. Em Associação Nacional de Pesquisa e Pós-Graduação em Psicologia (Eds.) (2002), Anais, VIII Simpósio de Pesquisa e Intercâmbio Científico (p.169 - 177). Serra Negra, SP: ANPEPP.

recebido em agosto de 2015

\section{Sobre as autoras}

Elizabeth do Nascimento é psicóloga e doutora em psicologia pela Universidade de Brasília. Professora associada do curso de graduação em psicologia e do Programa de Pós-graduação em psicologia do Departamento de Psicologia da Universidade Federal de Minas Gerais. Bolsista Produtividade do CNPq.

Alina Gomide Vasconcelos é psicóloga e doutora em psicologia pela Universidade Federal de Minas Gerais. Residente pós-doutoral do Programa de Pós-graduação em psicologia do Departamento de Psicologia da Universidade Federal de Minas Gerais. Psicóloga do Corpo de Bombeiros Militar de Minas Gerais. 